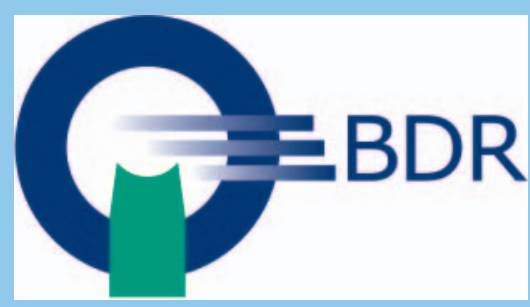

\title{
CIRSE 2019 - Passion - Culture - Care
}

Zum wiederholten Male fand die diesjährige Jahrestagung der Cardiovascular and Interventional Radiological Society of Europe (CIRSE) vom 7. bis 11. September in Barcelona statt. Bei ungebrochenem Zulauf aus aller Welt wurden erstmals mehr als 8000 registrierte Teilnehmer gezählt. Damit ist die Veranstaltung zum größten internationalen Podium für interventionelle Radiologie und bildgestützte Therapie geworden. Unabhängig davon, dass die katalanische Hauptstadt als Veranstaltungsort einen zusätzlichen Anziehungsmagneten darstellt und insbesondere für die aus nördlicheren Gefilden angereisten Teilnehmer eine willkommenen Verlängerung des Spätsommers darstellt, so ist dennoch die CIRSE-Jahrestagung eine Pflichtveranstaltung für interventionell tätige Ärzte weltweit. Erfreulicherweise nimmt auch beim CIRSE-Jahreskongress die Anzahl der Teilnehmer aus den Assistenzberufen deutlich sichtbar zu. Wie in den vergangenen Jahren fand auch 2019 zeit- und ortsgleich das Interdisciplinary Endovascular Aortic Symposium (IDEAS) statt. Und beschäftigte sich im Jubiläumsjahr der 25. Wiederkehr der ersten endovaskulär implantierten Aortenprothese erneut im interdisziplinären Format zwischen Gefäßchirurgen und interventionellen Radiologen mit dem aktuellen Stand der Therapie von Aortenerkrankungen.

Aus dem umfassenden und alle Bereiche der Interventionellen Diagnostik und Therapie abdeckenden Programm lassen sich fünf Hauptbereiche fokussieren: die erwähnte Therapie von Aortenerkrankungen, die Behandlung der PAVK, die interventionelle Tumortherapie, neue und neuere interventionelle Therapieverfahren sowie der Strahlenschutz in der bildgestützten Diagnostik und Therapie. Ohne den Anspruch auf Vollständigkeit seien einzelne High- lights aus den verschiedenen Bereichen herausgegriffen.

Auf dem IDEAS-Symposium wurde interessanterweise im Jubiläumsjahr in bisher nicht dagewesener Offenheit die endovaskuläre Versorgung von Aortenaneurysmata in Frage gestellt und auf die nach wie vor zur Verfügung stehende offene chirurgische Versorgung verwiesen. Hintergrund sind Studien, die Langzeitbeobachtungen mit einschließen und von späten bzw. sehr späten Komplikationen bis zur Ruptur der implantierten Prothese berichten. Man führt dies auf zunächst Mikroläsionen im Prothesenkörper zurück, die über Mikroblutungen bei längerem Bestehen jedoch zu letalem Ausgang führen können. Auch wenn die endovaskuläre Versorgung eines Aortenaneurysmas im Notfall oder bei instabilem Patienten in jedem Fall der offenen Versorgung vorzuziehen ist, sollte, nach Meinung zahlreicher internationale Experten, im elektiven Fall, bei stabilem und operablem Patienten die offene OP vorgezogen, ja zumindest erwogen werden. Dies umso mehr, wenn die anatomischen Verhältnisse am Prothesenhals schwierig sind und ggf. die Implantation einer fenestrierten Prothese notwendig machen. Zur Diskussion ob im Falle einer unzureichenden Landungszone am proximalen Prothesenende einer fenestrierten Prothese oder dem sogenannten Chimney-Verfahren der Vorzug zu geben ist, waren die Meinungen weiterhin unterschiedlich. Zu berücksichtigen sind dabei sicherlich - neben der Expertise des Teams - die Akuität der Versorgung. Dauert doch die Maßanfertigung einer fenestrierten Prothese einige Wochen. Berufspolitisch brisant ist die Diskussion in so ferne, als die Expertise zur offenen Aorten-OP unter jüngeren Gefäßchirurgen aufgrund des in der Regel angewandten endovaskulären Verfahrens vielfach annähernd verloren gegangen ist. Auch sind die Erfah- rungen mit komplizierten endovaskulären Spezialeingriffen kaum flächendeckend verfügbar. Die Stärkung der interdisziplinären, zertifizierten Gefäßzentren ist deshalb zum Wohle von Patienten und Operateuren - unbedingt weiter zu fördern.

Im Bereich der peripheren vaskulären Interventionen wurde die Diskussion hauptsächlich von der Frage beherrscht, inwieweit medikamentenbeladene Ballone (DEB) und Stents (DES) weiterhin anwendbar sind oder nicht. Basis der Diskussion ist die zum Jahreswechsel erschienene Metaanalyse, die zum Ergebnis kommt, dass in der Gruppe der mit medikamentenbeladenen Ballonen behandelten Patienten die Todesrate signifikant erhöht war. Auch wenn der Pathomechanismus aktuell nicht eindeutig geklärt ist, haben sich auch führende Repräsentanten in Barcelona für einen Stopp im Einsatz der DEBs stark gemacht. Umgekehrt gab es auch zahlreiche Stimmen erfahrener Anwender, die eine Anwendung weiterhin befürworten. Allerdings ist zu beachten, dass die Aufsichtsbehörden in unterschiedlichsten Ländern inzwischen Hinweise hierzu herausgegeben haben. Dabei wird die Verantwortung vollständig in die Hand des Anwenders übertragen ein Verbot von DEB und DES wurde bisher nicht ausgesprochen. Dies könnte jedoch erfolgen, wenn durch die Anwender nicht ausreichendes Datenmaterial zur Wiederlegung des im Raum stehenden Verdachts beigebracht wird. Sollte die Anwendung medikamentenbeladener Devices eingeschränkt oder abgeschafft werden ist mit erhöhten Rezidivraten, vermehrten Wiederholungseingriffen und erhöhten Gesamtkosten zu rechnen.

Aus dem Bereich der interventionellen Tumortherapie sei die Ablation von Nierentumoren herausgegriffen. Das Verfahren ist 
seit Jahren in unterschiedlichsten Zentren in der Anwendung. Dennoch fehlen standardisierte Daten zum geeigneten Verfahren, zum Vorgehen und zur Erfolgskontrolle. Dabei muss sich die Methode zunehmend mit roboterassistierten Methoden der urologischen Chirurgie messen. Diese sind allerdings deutlich aufwändiger und teurer. In verschiedenen Diskussionsrunden waren sich die Teilnehmer aus Urologie und interventioneller Radiologie einig, dass es dringend geboten ist auf Seiten der Radiologie eine Standardisierung herbeizuführen, die zwingend ein weitgehend einheitliches Verfahren - präferiert wird die Cryoablation eine prätherapeutische histologische Sicherung und das Erreichen einer R0-Situation post ablationem vorsieht. Andernfalls ist das Verfahren in Gefahr an Bedeutung zu verlieren. Auch hier ist eine Zentralisierung bzw. standardisierte flächendeckende Versorgung unbedingt anzustreben.

Seit vor ca. zwei Jahren die ersten interventionell anlegbaren Dialyse-Shunts vorgestellt wurden, haben sich die Applikationsverfahren deutlich verbessert. Jetzt steht ein auf 4F-Basis montiertes System zur Verfügung, das über die Radialarterie und die Radialvene unter Ultraschallkontrolle eingebracht wird und mittels Radio-FrequenzAblation eine arterio-venöse Fistel mit Punktionsmöglichkeit erzeugt. Das Verfahren überzeugt vor allem deshalb, weil der periphere Zugang deutlich komfortabler in der Anwendung ist für den Operateur als der Zugang über die Ellenbeuge. Limitierend sind wohl auch weiterhin die bei vielen Patienten dieses Klientels limitierten peripheren Gefäßdurchmesser.
Nicht erst seit der Revision der Strahlenschutzvorgaben auf europäischer Ebene ist das Thema Dosisminimierung, besonders auch in der interventionellen Radiologie angekommen. Dem hat die CIRSE durch eine weitere Aufstockung des Platzes und einer Neugestaltung des Radiation Protection Pavillions Rechnung getragen. Unter Radiologen ist die Problematik zweifelsohne sehr präsent, mehr Durchdringung würde man sich hierzu bei den Anwendern aus der Chirurgie wünschen.

Was wäre ein CIRSE-Kongress ohne die Industrieausstellung mit ihren Neuheiten und Weiterentwicklungen. Diese zeigten sich in diesem Jahr sowohl in der Geräteindustrie, als auch bei den Herstellern von Untersuchungsmaterialien. Insbesondere in großen Zentren lässt sich ein Trend zum interventionellen Eingriffsraum mit MehrebenenAngiografie und/oder CT-Möglichkeit erkennen. Angestrebt wird ein interdisziplinär genutzter interventioneller Arbeitsraum mit OP-Bedingungen, ausreichender Anästhesiemöglichkeit und ausgestattet für jegliche Form bildgestützter Therapieverfahren. In Zeiten limitierter Ressourcen sicherlich ein anzustrebender Weg wobei sich zentrumsbedingt Schwerpunkte herausbilden dürften. Inwieweit sich dieses Raumkonzept für die flächendeckende Versorgung mit interventionellen Verfahren anbietet, wird sich zeigen müssen. Angesichts der zunehmenden Bedeutung bildgestützter Therapieverfahren wird eine Spezialisierung, abhängig vom Spektrum des jeweiligen Standortes, erfolgen müssen um die Breite der verfügbaren Verfahren mit ausreichender Expertise anbieten zu können.
Im Rahmen der Mitgliederversammlung der CIRSE fand auch die Übergabe der Präsidentschaft von Robert A. Morgan (London) auf Afshin Gangi (Strassburg) statt. Damit ging eine erfolgreiche Präsidentschaft, die insbesondere durch eine starke Steigerung der Mitglieder der Gesellschaft und der Teilnehmer am Jahreskongress geprägt war zu Ende. Als Teilnehmer verlässt man Barcelona nach 5 Tagen Kongress mit einer Fülle neuer Eindrücke, der Bestätigung des eigenen Tuns in vielen Bereichen, aber auch der Erkenntnis der Notwendigkeit der permanenten Weiterentwicklung.

Das nächste Update hierzu gibt es auf der CIRSE-Tagung 2020 in München. Angesichts des Veranstaltungsortes im eigenen Land ein zusätzlicher Ansporn wieder mit dabei zu sein - und auch Kollegen und Mitarbeiter zu überzeugen sich dieses Angebot zu Information und Austausch nicht entgehen zu lassen.

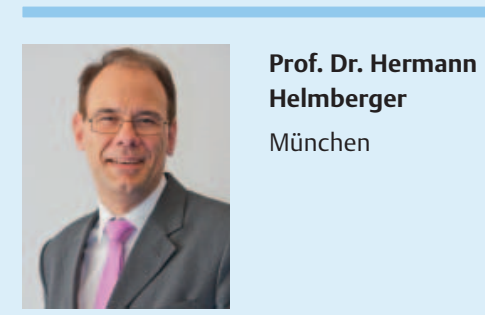

